\title{
Induced Heating in Steel-Beam Reinforced Ceilings over Three- Phase Transformer Delta Closures
}

\author{
Peter F. Ryff
}

Ryerson University

\section{Bulat}

EMPCO (Canada) Ltd.

Ryff, P. F., \& Bulat, V. (1980). Induced heating in steel-beam reinforced ceilings over three-phase transformer delta closures. IEEE Transactions on Power Apparatus and Systems, PAS-99(6), 2448-2452.

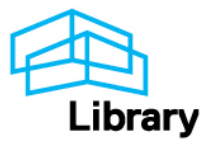




\author{
P.F.Ryff, Senior Member, IEEE \\ Ryerson Polytechnical Institute \\ Department of Electrical Technology \\ Toronto, Ontario, Canada
}

\author{
V. Bulat \\ EMPCo (Canada) Ltd. \\ Oshawa, Ontario, Canada
}

\begin{abstract}
Due to eddy currents, high temperatures often occur in structural parts adjacent to conductors carrying large currents. This paper deals with this problem and specifically with the minimum safe height and orientation of steel beams in a reinforced concrete ceiling above a three-phase transformer delta closure.

The power losses in the steel members due to induced eddy currents are calculated, from which the temperature increases are determined, properly accounting for convection and radiation effects. The methods presented are substantiated by experimental data and applied to a practical installation.
\end{abstract}

\section{INTRODUCTION}

The remarkable increases in power levels during the last few years of electric arc furnaces, used in steel-producing plants, also has produced many new problems. Not the least of them has been the heating of building steel adjacent to conductors carrying currents of up to $100 \mathrm{kA}$, in the event of Ultra High Power (UHP) furnace operation. Current ratings of existing furnaces being upgraded are increased by as much as 100 percent, sometimes without giving due regard to adjacent structural steel. One such instance, to be reported in this paper, was the question of proper spacing of a yetto-be-constructed reinforced concrete ceiling structure above a three-phase transformer delta closure as found in electric arc furnace installations. In the upgrading of the furnace steel output capacity, which among many other changes involves a larger capacity furnace transformer, the current levels were increased from $50 \mathrm{kA}$ to $80 \mathrm{kA}$. It became apparent that with these increased currents, greater consideration of induced heating effects in nearby structural steel was essential.

In addition to the economic value in minimizing these heating effects, there is also the question of stresses resulting from localized heating and the effects on the strength of the concrete and the bond between concrete and steel. Although the American Concrete Institute states that the strength and life of portland concrete is not inversely affected by temperatures as high as $120-260^{\circ} \mathrm{C}$, the bond between steel and concrete cycling at these elevated temperatures may well impose constraints on the

80 SM 543-9 A paper recommended and approved by the IEEE Transformers Committee of the IEEE Power Engineering Society for presentation at the IEEE PES Summer Meeting, Minneapolis, Minnesota, July 13-18, 1980. Manuscript submitted January 18, 1980; made available for printing April 22, 1980. design 1 . Hence, to evaluate critical design parameters and to maintain any induced heating well within this lower temperature limit and possibly to levels not to exceed those existing before upgrading (which were quite acceptable), this investigation was initiated. Particular emphasize was placed on determining the new ceiling height and steel beam orientation with respect to the current conductors. in view of induced heating and minimizing local surface temperatures. After presentation of the general theory and verification of calculated results by experimental data, the theory is applied to the practical installation considered.

\section{FORMULATION OF THE PROBIEM}

The configuration examined is illustrated in figure 1 , showing the secondary bus bars connecting the flexible cables to the threephase transformer secondary terminals. The reinforced ceiling and the to-be-determined distance $d$, with respect to the center phase, is also shown. The bus bars (two per phase) protrude through the furnace transformer wall in a triangulated manner, as shown in figure 2 . For analytical treatment two extreme and distinct situations are considered. In the first one, the steel structural beams run perpendicular to the secondary bus bars, while in the second, they are parallel to them. They will be examined in succession after which the general heat theory to calculate the temperatures is presented, which is common to both.

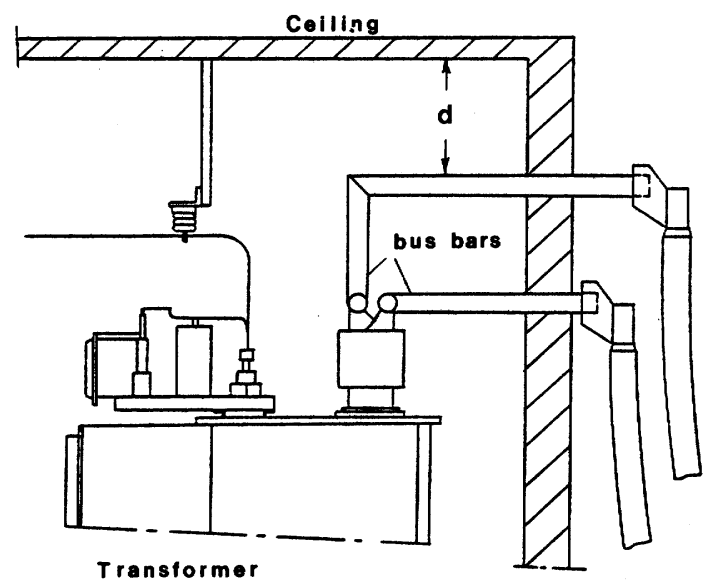

Fig. 1. Illustration of basic configuration.

\section{Perpendicular Geometry}

The basic arrangement considered is illustrated in figure 3. If it is assumed that there are many beams in close proximity relative to the distances of the geometry considered, the ceiling may be considered as a magnetic plate 


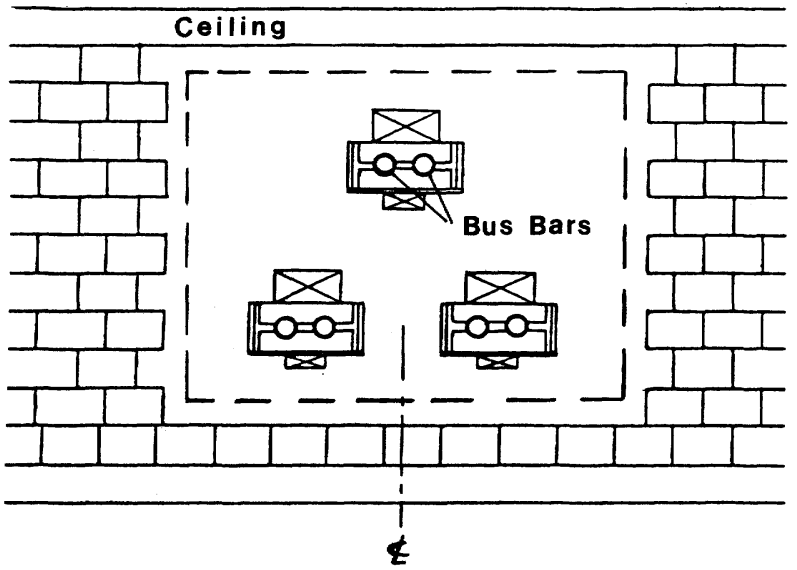

Transformer

Fig. 2. Showing triangulation of secondary bus bars through transformer wall.

for all practical purposes. This then will represent a worst possible situation from an induced heat point of view. The assumption seems well justified particularly when the concrete is further reinforced with rebars.

If at first a single conductor carrying a current I is considered, placed at a distance d, parallel to a magnetic plate of permeability $\mu=\mu_{0} \mu_{r}\left(\mu_{0}=4 \pi \times 10-7 \mathrm{H} / \mathrm{m}\right)$, it can be shown that the expression for the magnetic vector potential inside the material is 2 .

$$
\bar{A}=\frac{\mu I}{\pi} \int_{0}^{\infty} \frac{e^{-k d}}{k u_{r}+\gamma} e^{-\gamma y} \cos k x d k
$$

where $\gamma=\left(k^{2}+i \omega \mu \sigma\right)^{\frac{1}{2}}$

$\omega=2 \pi f$, the operating frequency

$\sigma=$ electrical conductivity $(\mathrm{s} / \mathrm{m})$

$\begin{aligned} \mu_{r} & =\text { relative permeability } \\ i_{-1} & \end{aligned}$

By extending the analysis to the geometry shown in figure 3 and using the superposition principle and notation of variables indicated, there results,

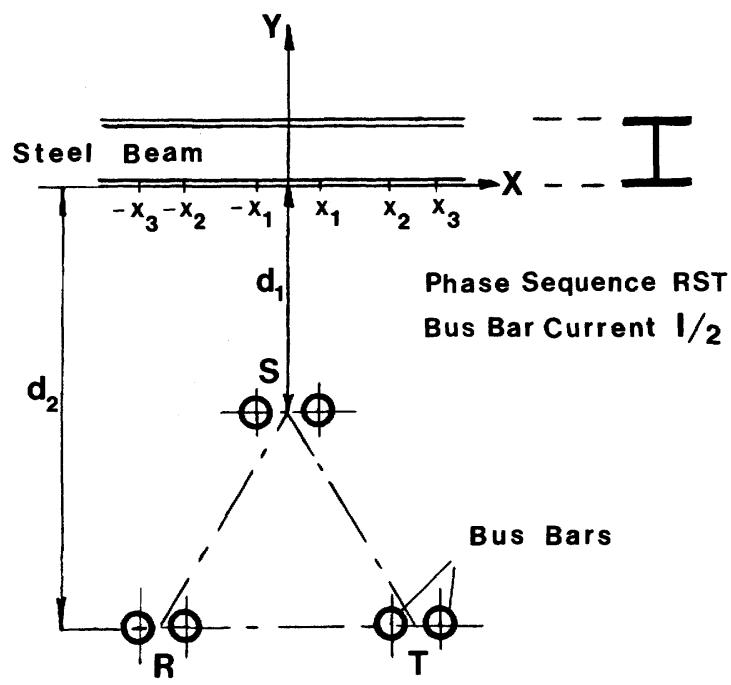

Fig. 3. Nomenclature for perpendicular geometry of beams relative to bus bars.

$$
\bar{A}=\frac{\mu I}{2 \pi} \int_{0}^{\infty} \frac{e^{-\gamma y}}{k \mu_{r}+\gamma} \cdot\left\{K_{1} e^{-k d_{1}}+\left(K_{2}+K_{3}\right) e^{-k d_{2}}\right\}
$$

in which,

$$
\begin{aligned}
& K_{1}=\left[\operatorname{cosk}\left(x-x_{1}\right)+\cos k\left(x+x_{1}\right)\right] \cdot\left(-\frac{1}{2}-i \frac{\sqrt{3}}{2}\right) \\
& K_{2}=\left[\operatorname{cosk}\left(x-x_{2}\right)+\cos k\left(x-x_{3}\right)\right] \cdot\left(-\frac{1}{2}+i \frac{\sqrt{3}}{2}\right) \\
& K_{3}=\operatorname{cosk}\left(x+x_{2}\right)+\cos k\left(x+x_{3}\right)
\end{aligned}
$$

The $x$-coordinates of the bus bar locations are denoted by the subscripted parameter $x_{j}$.

Equation (2) describes the vector potential in the material region. The current $I$ is the phase current being the total carried by the two busses per phase. Knowing the vector potential, the power loss density per unit volume $P_{v}$ can be obtained from,

$$
P_{v}=\sigma|\bar{E}|^{2}=\sigma|i \omega \bar{A}|^{2}=\sigma \omega^{2}|\bar{A}|^{2} \quad \omega / m^{3}
$$

\section{Parallel Geometry}

In the event that the structural beams run parallel to the three-phase conductor arrangement, the following derivation is appropriate. Here it is not assumed that the ceiling is comprised of a magnetic plate, but rather, it treats a single beam since it is assumed that adjacent beans are located such that their influence can be ignored. Also, because of the distances involved compared to the dimensions of the beam, it is assumed to be circular. It should be noted that this assumption represents a least-induced power loss geometry, compared to the above, which represented the worst possible condition.

These extreme situations were investigated since it was felt that having this knowledge any other possible ceiling construction must result in induced-heats which must fall within these outer limits. Hence, any design can be quickly assessed.

As in the previous analysis, a single conductor carrying a current $I$ and placed at a distance d from the circular beam is examined first, after which the resulting expression is again extended to apply to the arrangement depicted in figure 4. For the single conductor adjacent to a circular beam of radius $a$, it has been shown that the vector potential in the beam material is given by ${ }^{3}$.

$$
\bar{A}=-\frac{\mu I}{\pi} \sum_{n}\left(\frac{a}{d}\right)^{n} \frac{J_{n}(k \rho)}{n\left(\mu_{r}+1\right) J_{n}-k a J_{n+1}} \cos n \varphi(4)
$$

where $J_{n}$ is the Bessel function with argument $\mathrm{ka}$ if not specified, $\mathrm{k}^{2}=\omega u \sigma$ and $\rho$ and $\varphi$ are the cylindrical coordinates specifying the field point inside the beam where the potential is determined. All other parameters are as explained above. Applying the superposition principle and extending the analysis to the six bus-bar configuration of figure 4 , the resulting expression using the nomenclature indicated becomes 4 ,

$$
\begin{aligned}
A= & -\frac{\mu I}{2 \pi} \sum_{n} \frac{J_{n}(k \rho)}{n\left(\mu_{r}+1\right) J_{n}(k a)-k a J_{n+1}(k a)} . \\
& \cdot\left\{\left(\frac{a}{d_{1}}\right)^{n} \cos n\left(\varphi+\theta_{3}\right)+\left(\frac{a}{d 2}\right)^{n} \cos n\left(\varphi+\theta_{2}\right)+\right.
\end{aligned}
$$




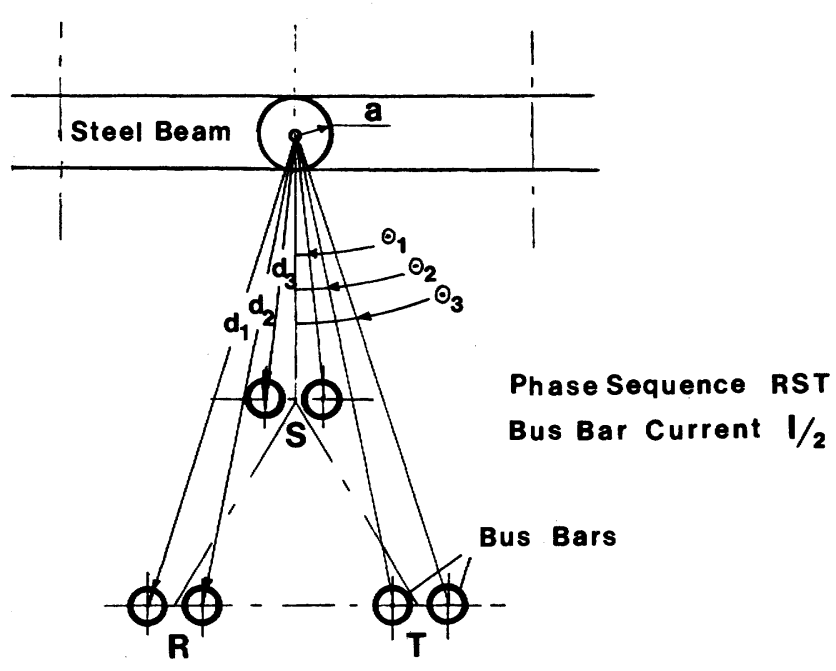

Fig. 4. Nomenclature for parallel geometry of beams relative to bus bars.

$+a_{1}\left[\left(\frac{a}{d_{3}}\right)^{n} \cos n\left(\varphi+\theta_{1}\right)+\left(\frac{a}{d 3}\right)^{n} \cos n\left(\varphi-\theta_{1}\right)\right]$

$\left.+a_{2}\left[\left(\frac{a}{d_{2}}\right)^{n} \cos n\left(\varphi-\theta_{2}\right)+\left(\frac{a}{d_{1}}\right)^{n} \cos n\left(\varphi-\theta_{3}\right)\right]\right\}$

which represents the contribution of each bus bar carrying half its corresponding phase current, The phase relation of the supply current is accounted for by the constants,

$$
a_{1}=-\frac{1}{2}-i \frac{\sqrt{3}}{2} \text { and } a_{2}=-\frac{1}{2}+i \frac{\sqrt{3}}{2}
$$

The power 1088 density inside the material is again obtained by using equation ( 3 ).

\section{Temperature Calculation}

Having determined the power 1088 density throughout the material region in both cases. the corresponding temperature effect remains to be found. For this the induced power 1088 density at the surface where the temperature is to be calculated is obtained by the methods presented. As mentioned before, the thickness 1 of the plate is assumed large for electrical but small for thermal calculations.

The induced power 10sses, $q^{m+}=q_{0}^{\prime \prime \prime} e^{-x / \delta}$. represents the internal heat generation term in the thermal calculations ( $q_{0}$ being the value at the surface) which decays exponentially into the material. Even in the event of a single I-beam, the web thickness is very small compared to the flange dimensions, hence, it is assumed that in the thermal calculations only the flange need to be considered, which in effect can then also be treated as a plate.

Referring to figure 5, the equation governing the temperature distribution $T(x)$, in the plate (ceiling) material is described by,

$$
\frac{d^{2} T(x)}{d x^{2}}+\frac{q^{\prime \prime \prime}(x)}{k}=0
$$

with the boundary conditions applying to the top and bottom surface of the plate material, being 5 . at $x=0, \quad k \frac{d T(0)}{d x}=h_{b}\left[T(0)-T_{b}\right]$

and $x=1,-k \frac{d T(1)}{d x}=h_{t}\left[T(1)-T_{t}\right]$

where $k=t h e r m a l$ conductivity of the material. $T_{t}$ and $T_{b}=a m b i e n t$ temperature above and below the celling, respectively. $h_{t}$ and $h_{b}=$ surface heat transfer coefficient accounting for convection and radiation at the top and bottom surface, respectively 6 .

Equations (6), (7) and (8) completely describe the temperature distribution in the material for steady-state conditions. The resulting expression, obtained after a somewhat lengthy but straightforward algebraic manipulation, gives the temperature throughout the material and is of the form?.

$$
T=-\phi \delta e^{-x / \delta}+c_{1} x+c_{2}
$$

where $c_{1}=\frac{-\phi \beta-h_{b} h_{t}\left(T_{b}-T_{t}\right) / k}{h_{t}+h_{b}\left(1+h_{t} 1 / k\right)}$

$c_{2}=\frac{\phi \beta+h_{b}\left(1+h_{t} I / k\right) T_{b}+h_{t} T_{t}}{h_{t}+h_{b}\left(1+h_{t} l / k\right)}$

and $\quad \alpha=h_{t}\left(1+h_{b} \delta / k\right)+h_{b}\left(1-h_{t} \delta / k\right) e^{-1 / \delta}$

$$
\begin{aligned}
& \beta=\left(k+h_{b} \delta\right)\left(1+h_{t} 1 / k\right)-\left(k-h_{t} \delta\right) e^{-1 / \delta} \\
& \phi=\frac{\delta q_{0}^{\prime \prime \prime}}{k}
\end{aligned}
$$

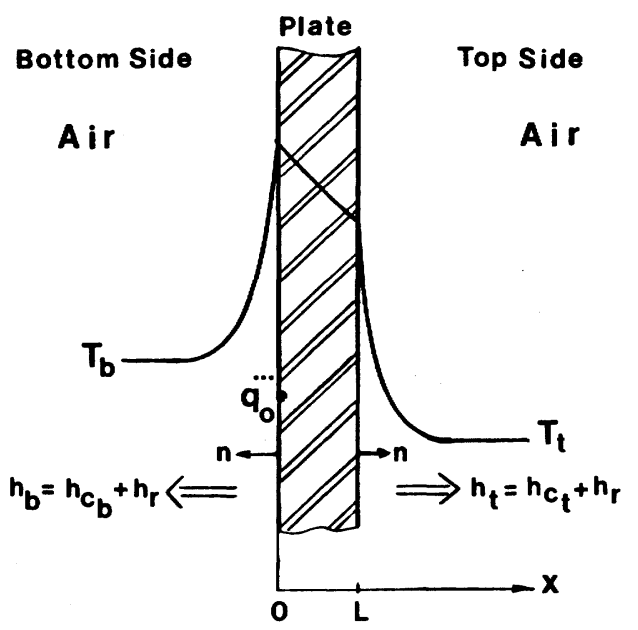

Fig. 5. Sketch showing nomenclature for heat conduction in plate.

\section{Verification of Methods Presented}

Using the methods presented, the power loss density and resulting temperature at the surface of the plate material was calculated as a function of the distance, for the two conductor 
arrangements shown in figures 6 and 7 . These examples were chosen since extensive published experimental data by Swerdlow and Buchta1 could be compared with these calculations. It can be seen in both instances that they compare very well, thereby substantiating the theories presented. At the smaller distances it can be seen that the results of the two methods converge, as expected, since even in the event of a single beam its dimensions become large compared to the distances involved. It is also interesting to note that the calculated results for the single beam are somewhat smaller and for the plate somewhat larger than the experimental results, respectively. For the ceiling consisting of a plate it is as expected. However, for the single beam these small deviations are expected to be caused by slight variations in material constants used in the calculations.

\section{Actual Installation}

The existing installation as depicted in figures 1 and 2 , has the secondary bus-bars in a triangulated geometry measuring $4^{\prime}-1^{\prime \prime}$. the current per phase is $60 \mathrm{kA}$. The two busses per phase are spaced at $9.75^{\circ}$ with a ceiling height of $6^{\prime}-6^{\prime \prime}$ above the center-phase. The constructional beams are in a parallel geometry and widely spaced compared to the dimensions in question. Using the method for the parallel geometry the surface temperatures were calculated for a range of distances, shown in figure 8 , by curve $i$. The maximum measured temperature (actual temperature will fluctuate depending on the particular "load" condition in the furnace) was approximately 35 oC, being close to the predicted value at the distance involved.

Maintaining the same triangulation of the bus-bars but increasing the phase current to $80 \mathrm{kA}$ (nominal) and recalculating the tempera-

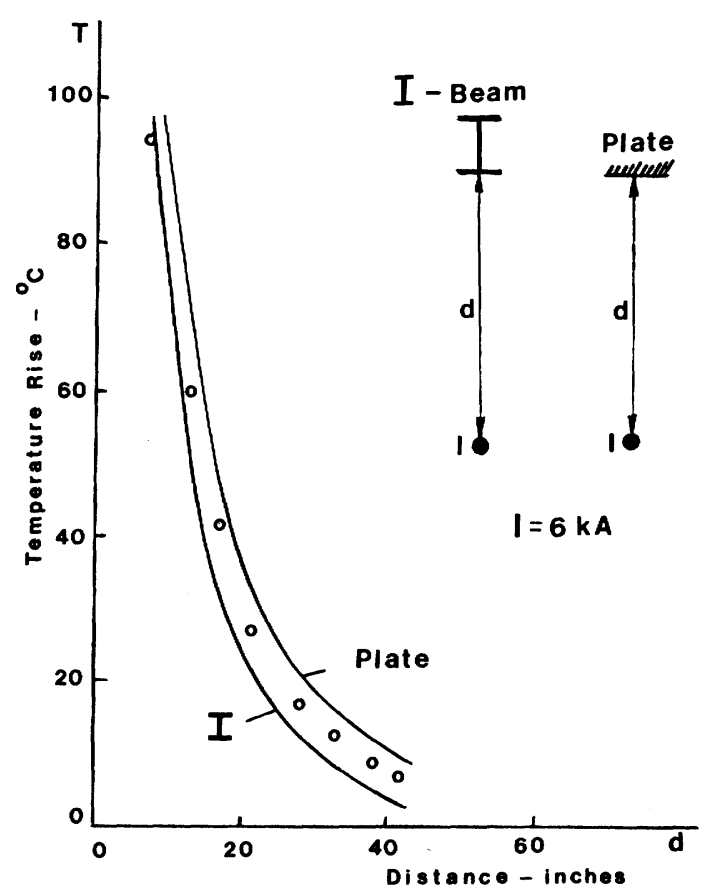

Fig. 6. Temperature rise at the surface due to a single conductor. Circles show experimental values for I-beam. tures, produced curve $i \mathrm{i}$ in figure 8 . Using the method for the "plated" ceiling with this phase current resulted in curve iii. As expected, these temperatures are considerably higher. Therefore, to maintain the induced losses (and subsequent temperature increases) at the increased current levels to those existing before upgrading, it was recommended to run beams in parallel to the secondary bus-bars at a minimum ceiling height of $7^{\prime}-6^{\prime \prime}$. The new ceiling presently being installed is constructed according to these recommendations.

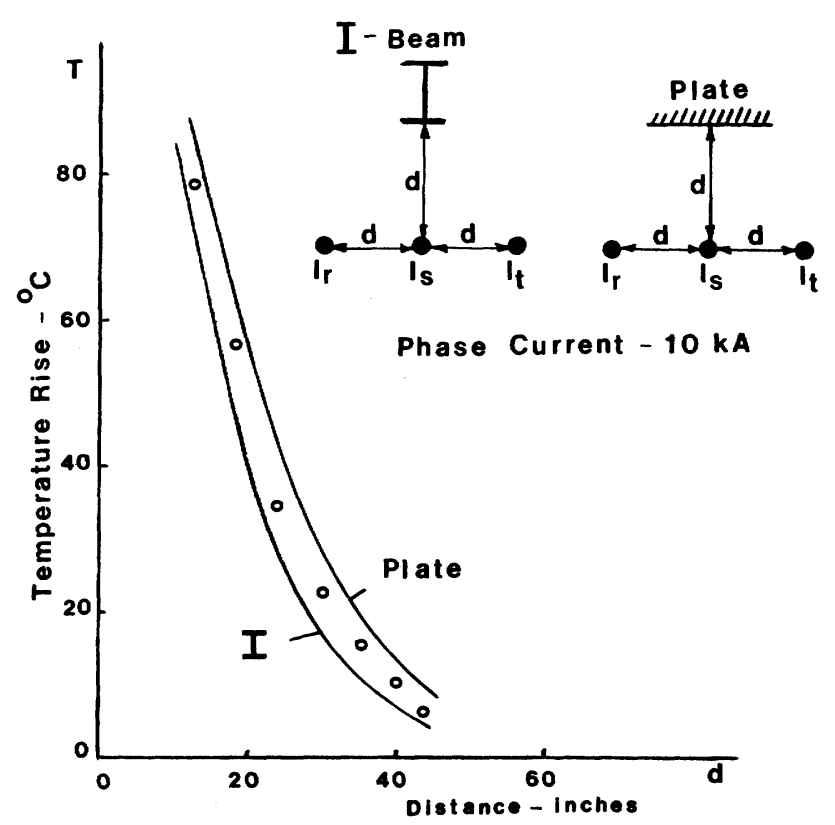

Fig. 7. Temperature rise at the surface due to three-phase conductors. Circles show experimental values for I-beam.

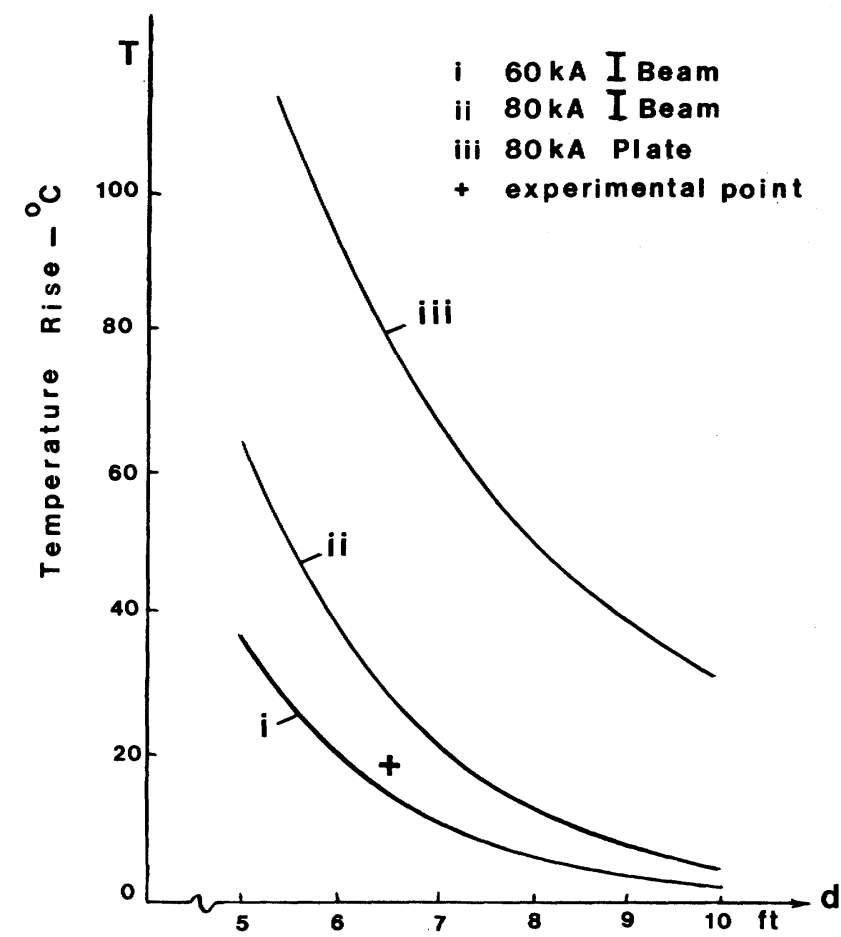

Fig. 8. Surface temperature increases in actual installation (+ indicates measured value in existing installation). 


\section{CONCLUSIONS}

The calculated results have been substantiated by experimental data in a variety of conductor geometries and ceiling constructions. The agreement in all situations is excellent thereby substantiating the theories presented.

\section{REFERENCES}

(1) N.Swerdlow and M.A.Buchta. "Practical Solutions of Inductive Heating Problems Resulting from High Current Busses". IEEE Trans. PAS-78, pg. 1936, October 1960.

(2) Richard I.Stoll. The Analysis of Eddy Currents. Clarendon Press, Oxford 1974, pg.63.

(3) Milos Stafl. Electrodynamics of Electrical Machines. Iliffe, London, 1967. pg. 151.

(4) P.F.Ryff. "Eddy Currents in Cylindrical Arc Furnace Shells". Paper submitted for publication to the IAS (EPH-Committee), October 1979.

(5) Vedat Arpaci. Conduction Heat Transfer. Addison-Wesley Publishing Co. , 1966, pg. 128.

(6) W.H.McAdam. Heat Transmission. McGraw-Hill Book Co.., New York 1954, Third Edition, pg. 180 .

(7) P.F.Ryff and V.Bulat. "Carbon Steel versus Stainless Steel in the Design of Electrode Arms for UHP Electric Arc Furnaces". Paper submitted for publication to the IAS (EPHcommittee). December 1979.

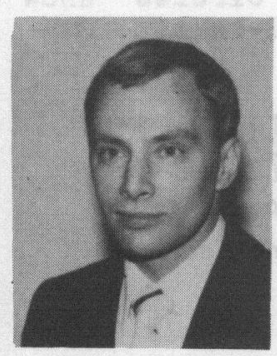

Peter F.Ryff $\left(s^{\circ} 66-M^{\prime} 66-\mathrm{SM}^{\circ} 78\right)$ was born in Amsterdam, The Netherlands. He received the Ingenieur degree in electrical engineering from the Engineering Collage in Amsterdam, The Netherlands, in 1958, and the B.A.Sc., M.A.Sc, and Ph.D. degrees in electrical engineering from the University of Toronto, Toronto, On., Canada, in 1966, 1967 and 1969 , respectively.
From 1958 to 1964 he was employed by Honeywell, Inc.. Toronto, On., and became their Engineering Field Representative. From 1969 to 1970 he was with General Time of Canada, Peterborough, On., as a Research Engineer in the field of electromagnetic and solid state time-base components. In 1970 he joined Ryerson Polytechnical Institute, Toronto, where he is Professor in the Electrical Technology Department. His research interests are in the fields of induction heating and arc melting furnaces.

Dr. Ryff is a member of the Association of Professional Engineers of the Province of ontario and the American Society for Engineering Education.

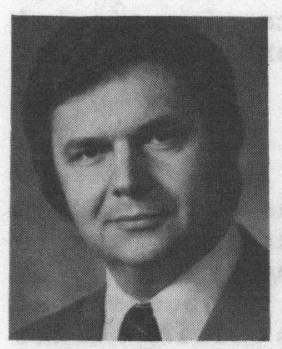

Vladimir Bulat received the B.S. degree (1960) and the M.S. degree (1973) in electrical engineering from the University of Zagreb, Yugoslavia. From 1960 to 1964 he was employed by a Yugoslavian company engaged in the design and installation of electric power distribution systems for industrial plants. From 1964 to 1974 he was with Zeljezara Sisak, Yugoslavia, where he held various engineering and management positions relating to electrical maintenance and electronic data processing. As the assistant to the Superintendent of electrical maintenance for the entire plant, he was involved in all areas of electrical design, planning, maintenance and system protection of the steel plant. From 1971 on he was their systems - programmer and then became Chief systems analyst for Technical applications. From 1974 to 1977 he was with Ferrco Engineering Itd.. Whitby, Ontario, where he was their Power Systems Engineer engaged in power systems analysis and flow studies, protective device coordination and design of distribution systems for mini-steel plants. He joined EMPCO (Canada) Itd. in 1977 as their Chief Electrical Engineer where he continued his activities in power systems analysis, feasibility studies, and proposal and equipment specifications in all areas of electric arc furnace designs and operations.

Mr. Bulat is a member of the Association of Professional Engineers of the Province of Ontario. 